\title{
Study protocol: rehabilitation including social and physical activity and education in children and teenagers with cancer (RESPECT)
}

Troels Thorsteinsson ${ }^{1}$, Anne Sofie Helms ${ }^{1,2}$, Lis Adamsen ${ }^{3}$, Lars Bo Andersen ${ }^{4}$, Karen Vitting Andersen ${ }^{1}$, Karl Bang Christensen ${ }^{5}$, Henrik Hasle ${ }^{6}$, Carsten Heilmann', Nete Hejgaard ${ }^{1,7}$, Christoffer Johansen ${ }^{8,9}$, Marianne Madsen ${ }^{1}$, Svend Aage Madsen ${ }^{1}$, Venka Simovska ${ }^{10}$, Birgit Strange ${ }^{11}$, Lone Friis Thing ${ }^{12}$, Peder Skov Wehner ${ }^{13}$, Kjeld Schmiegelow ${ }^{1,2}$ and Hanne Baekgaard Larsen ${ }^{1 *}$

\begin{abstract}
Background: During cancer treatment children have reduced contact with their social network of friends, and have limited participation in education, sports, and leisure activities. During and following cancer treatment, children describe school related problems, reduced physical fitness, and problems related to interaction with peers.

Methods/design: The RESPECT study is a nationwide population-based prospective, controlled, mixed-methods intervention study looking at children aged 6-18 years newly diagnosed with cancer in eastern Denmark $(n=120)$ and a matched control group in western Denmark $(n=120)$. RESPECT includes Danish-speaking children diagnosed with cancer and treated at pediatric oncology units in Denmark. Primary endpoints are the level of educational achievement one year after the cessation of first-line cancer therapy, and the value of $\mathrm{VO}_{2 \max }$ one year after the cessation of first-line cancer therapy. Secondary endpoints are quality of life measured by validated questionnaires and interviews, and physical performance. RESPECT includes a multimodal intervention program, including ambassador-facilitated educational, physical, and social interventions. The educational intervention includes an educational program aimed at the child with cancer, the child's schoolteachers and classmates, and the child's parents. Children with cancer will each have two ambassadors assigned from their class. The ambassadors visit the child with cancer at the hospital at alternating 2-week intervals and participate in the intervention program. The physical and social intervention examines the effect of early, structured, individualized, and continuous physical activity from diagnosis throughout the treatment period. The patients are tested at diagnosis, at 3 and 6 months after diagnosis, and one year after the cessation of treatment. The study is powered to quantify the impact of the combined educational, physical, and social intervention programs.

Discussion: RESPECT is the first population-based study to examine the effect of early rehabilitation for children with cancer, and to use healthy classmates as ambassadors to facilitate the normalization of social life in the hospital. For children with cancer, RESPECT contributes to expanding knowledge on rehabilitation that can also facilitate rehabilitation of other children undergoing hospitalization for long-term illness.
\end{abstract}

Trial registration: Clinical Trials.gov: file. NCT01772849 and NCT01772862

Keywords: Cancer, Pediatric, Children, Rehabilitation, Physical activity, Quality of life, Intervention, Peers, Controlled, School reentry

\footnotetext{
* Correspondence: hanne@baekgaard.com

1 Department of Pediatrics and Adolescent Medicine, Copenhagen University Hospital Rigshospitalet, Blegdamsvej 9, 2100 Copenhagen, Denmark

Full list of author information is available at the end of the article
} 


\section{Background}

Each year 200 children and adolescents in Denmark are diagnosed with cancer. Over recent decades the increased understanding of cancer biology, improved surgery and chemotherapy, and generally intensified treatment, have resulted in 5-year survival rates above 80\% [1-3]. However, the disadvantages of this improvement include severe acute and late effects [2-4], often involving isolation for long periods of time, both when in hospital and at home. This isolation reduces participation in activities with peers at school and in sporting activities $[1,5,6]$. As a result, important social interactions and the natural development of social skills with classmates are disrupted $[5,6]$. Following diagnosis, children are absent from school for an average of 85 days [7] during the first 12 to 18 months, and 3 years after diagnosis their school attendance is still irregular and many fail classes $[8,9]$. Childhood and adolescent cancer survivors report being bullied, feeling isolated [10-13], and having few or no friends [14-16]. Although register-based data show that they achieve the expected educational level post-treatment [17], such data do not address social and physical functioning [18-20]. Attending school may help provide normality, continuity, and security, in an abnormal life situation [21]. Few intervention studies have addressed children's reentry into school during and following treatment. Being able to participate in normal school activities with peers includes being physically active. However, very few studies have been published on physical activity in children with cancer [22-24]. These studies have in general been burdened by the diversity of exercise and outcome measurements, and/ or limited duration of the interventions [22,24-26]. Overall, the published studies have shown that children with cancer are less physically active and have decreased muscle strength, balance, and cardiovascular condition compared with peers, both during and following treatment $[8,16,26,27]$. The few studies carried out during treatment show that it is possible to improve children's physical functioning, both during and following treatment [23].

The burden of the disease, and body modifications resulting from treatment and reduced physical activity, may lead to lower self-esteem and emotional well-being, and compromised social relationships, which negatively influence the quality of life for children with cancer $[12,13,28,29]$. However, it is unclear to what extent this is related to the disease and treatment burden, the child's learning difficulties, physical decline, or changes in social position [30-32].

Importantly, none of the intervention studies address the potential effects of early rehabilitation from the time of diagnosis as a tool to maintain the children's social network during treatment [28,30,32]. The Rehabilitation including Social and Physical activity and Education for
Children and Teenagers with Cancer (RESPECT) study is inspired by Erving Goffman's symbolic interaction theory [33-35], Thomas Scheff's theory and concepts of emotional and social bonds [36,37], and Venka Simovska's definition of interactive processes and empowerment [38].

The overall purpose of the RESPECT study is to examine whether involving healthy classmates at the hospital from the time of diagnosis and throughout treatment will improve the educational, physical, and social performance of children with cancer and facilitate their reentry into everyday life following treatment.

\section{Methods \\ Trial design}

RESPECT is a multimodal intervention program for children undergoing cancer treatment. This study is an integrated part of a newly established comprehensive rehabilitation program (CIRE) for children and adults during and following their cancer diagnosis. The overall aim of the CIRE program is to identify rehabilitation needs, apply early physical training, and to combine quantitative and qualitative research methods to understand the functional, cognitive, emotional, social, and physiological mechanisms involved in successful rehabilitation.

\section{Participants}

Children aged 6-18 years diagnosed with cancer and treated with chemotherapy/irradiation, or diagnosed with Langerhans cell histiocytosis (LCH) or myelodysplastic syndrome (MDS) and treated with chemotherapy, at any pediatric oncology unit in Denmark are eligible for the study. All participants are enrolled at school at the time of diagnosis and are able to communicate in Danish. Children with mental disability (e.g. Down syndrome), severe co-morbidity, or terminal illness at the time of diagnosis are excluded. Patients diagnosed in the period 2013-2015 at the Copenhagen University Hospital Rigshospitalet $(n=120)$, are assigned to the intervention group.

Four control groups are identified: 1) children with cancer diagnosed at Odense University Hospital, Aarhus University Hospital, and Aalborg University Hospital in the period 2013-2015 $(n=120)$. Secondary control groups are 2) the sibling closest in age (regardless of gender) to the child with cancer, and 3) the child's classmates. In addition, we include 4) a historical nationwide control group of children with cancer treated from February to December 2012 ( $\mathrm{n}=113$ families).

\section{Interventions}

\section{Educational intervention}

The educational intervention includes a 90-minute presentation on cancer given at the child's school and aimed at the child's classmates. The information included covers the etiology of childhood cancer, the specific subtype of 
cancer, the treatment, the expected side effects, supportive care, everyday life at the hospital, communication and emotional strains, the importance of physical activity during treatment, and the role of the ambassadors. The teacher develops a weekly-updated curriculum, which the child is to follow in cooperation with the hospital schoolteachers and the ambassadors. At the meeting a consent form to be completed at home is handed out, on which all parents indicate whether their child is capable of acting as an ambassador for the classmate with cancer. In collaboration with the class teacher, the children with cancer, the families of the classmates, and the RESPECT research team, two ambassadors are selected. In this way the children with cancer each have two ambassadors assigned from their class. The ambassadors alternately visit the child with cancer when at hospital, at least twice monthly, throughout the treatment period. The ambassadors visit the pediatric oncology ward involved in the patient's treatment and participate in the hospital school program, share meals in the kitchen, and participate in physical and social activities. The ambassadors therefore act as a bridge between the child's everyday life at home and at the hospital, and serve as role models. Furthermore, the ambassador provides moral support, familiarity, and encouragement, and helps to create a friendly educational, physical, and social environment for the child with cancer. By involving healthy children, we will examine whether the creation of a more normal everyday life during treatment can reduce stigmatization of children with cancer and facilitate rehabilitation following treatment. The intervention program will be active in the periods during treatment when the child is attending their regular school for less than 3 days a week, 4 hours per day.

\section{Physical and social intervention}

The supervised hospital-based physical and social activity program from diagnosis and throughout treatment includes daily participation in an individual training scheme and participation in joint physical and social activities twice a week during hospitalization. As the literature shows [23], we cannot reliably quantify the effect of a home-based training program, therefore the program is intended for physical activity in a hospital setting. The physical activity intervention focuses on muscle strength, cardio-respiratory fitness, and balance. Individual training sessions take place 3 to 5 days a week and training sessions vary from 5-120 minutes per session, depending on the type of training and the general condition of the child. The twice-weekly group training includes all study patients and ambassadors at the hospital on that specific day.

\section{Primary endpoints}

\section{Educational intervention}

The primary endpoint of the educational intervention is the child's level of school achievement one year after cessation of first-line cancer treatment. The school achievement includes the child's level of education in language, reading and writing skills, and mathematics. It is measured on a five-point response scale (outstanding level of performance, high level of performance, satisfactory level of performance, needs improvement in level of performance, and unsatisfactory level of performance).

\section{Physical and social intervention}

The primary endpoint of the physical and social intervention is the level of $\mathrm{VO}_{2 \max }$, determined during exercise testing on a cycle ergometer after the Godfrey protocol [39], one year after the cessation of first-line cancer treatment.

\section{Secondary endpoints}

The secondary endpoints include both quality of life and physical performance. We hypothesize that the components in the intervention will enhance the children's quality of life 6 months after diagnosis and one-year post treatment. Quality of life is assessed using validated questionnaires and interviews.

\section{Outcome measures}

The effects of the intervention program are quantified using validated questionnaires, physical fitness tests, blood tests, and full body dual energy X-ray absorptiometry (DEXA scan).

This comprehensive monitoring takes place at diagnosis, 3 and 6 months from baseline, one year after the cessation of treatment, and the long term effects of the program will continue to be monitored every 5 years.

\section{Questionnaires}

The PedsQL Core [40] measures the quality of life in children using 23 questions on a five-point response scale from never to almost always. The answers are divided into four domains: health and physical activity, emotions, dealing with others, and school activity.

The Strength and Difficulties Questionnaire (SDQ) [41] consists of two parts. Part one includes 25 questions on a three-point response scale from does not fit to fits well in the following five areas: emotional symptoms, behavior, hyperactivity and concentration problems, problems with peers, and pro-social behavior. If the respondent confirms problems with concentration, behavior, or interaction with others, then part two of the questionnaire examines the duration, severity, extent, and social impact on the environment using a four-point response scale, from not at all to very much. 
The Revised Child Anxiety and Depression Scale (RCADS) [42] measures anxiety and depression using 47 questions on a four-point response scale from not at all to very often, according to the DSM-IV criteria. The RCADS includes the following subscales: separation anxiety, social phobia, generalized anxiety disorder, panic disorder, obsessive thoughts and actions, and depression.

Resilience [43] measures resilience as personal competence, social competence, ability to maintain structure, family cohesion, and social support based on 28 questions on a five-point response scale from strongly agree to strongly disagree.

The Loneliness and social Dissatisfaction Questionnaire (LDQ) [44] includes 24 questions to assess loneliness, social dissatisfaction, and hobbies on a three-point response scale with the answer categories no, sometimes, and yes.

The Children's Impact of Event Scale 13 [45] measures problems which the child or adolescent may experience after a stressful event using 13 questions on a four-point response scale from not at all to often. It contains three subscales: intrusion, avoidance, and arousal.

The best friend nominations scale [46] asks the child with cancer and his or her classmates to nominate their two best friends in the class. This provides a standardized total score for the number of nominations that each child receives and the mutual friendship score shows how many of their friendship choices are reciprocated. This is examined at the time of diagnosis and one year after treatment. The best friend nomination scale is disguised as a friendly exercise in the class, and not directly related to the child with cancer.

\section{Self-generated questionnaires}

Self-generated questionnaires are used to record demographics, school participation, ambassador participation, the thoughts and reflections of schoolteachers at the time of diagnosis, evaluation of the educational sessions in class, academic position statements from teachers, physical activity before diagnosis, and physical training at home.

\section{Physical tests}

The effect of the physical training program is assessed based on physical strength, balance, and a fitness test. The Andersen test [47] measures fitness using a 10minute run or other high energy moving activity in intervals of 15 seconds of activity and 15 seconds of rest. The distance after 10 minutes and maximum and average heart rate is measured. The $\mathrm{VO}_{2 \max }$ is measured during a cycle ergometer test with the workload increasing progressively to the point of exhaustion [48] using a Hans Rudolph valve (2-way NRBV, Hans Rudolph Inc., Kansas City, MO, USA). The timed Up-and-Go test
[49] tests basic mobility, defined as the ability to get in and out of bed, to get up and down from a chair, to walk short distances, and to turn. The test measures the time it takes to get up from a chair with armrests, walk 3 meters, turn, walk back to the chair and sit down again. The sit-to-stand test [50] evaluates muscle strength in the lower extremities and in the hip and core muscles. The test measures the number of repetitions of getting up from a sitting position to standing fully upright and sitting down again completed in 30 seconds. Hand strength as an indication of manual force is measured using a handheld dynamometer [51]. Two trials are conducted for each arm and are performed standing or sitting but with the elbow and dynamometer not touching anything. The flamingo balance test [52] measures the ability to balance on one leg and provides information about leg, hip, and abdominal muscle strength. The child is barefoot and balances on their preferred leg with the opposite leg lifted from the ground. The number of times the child loses balance in 60 seconds is registered. Each time the child loses balance the clock is stopped and then restarted when the child is ready.

Children in the intervention group and children in the control group diagnosed and being treated for cancer at Odense University Hospital, Aarhus University Hospital, and Aalborg University Hospital in the period 20132015, perform the physical tests.

\section{Statistical considerations I}

We expect all children aged 6-18 years diagnosed with cancer in Denmark during 2013-2015 will be included in the study. The primary endpoint for school achievement is classified using five ordinal categories: outstanding level of performance, high level of performance, satisfactory level of performance, needs improvement in level of performance, and unsatisfactory level of performance, with the frequency distribution of these five categories being $10 \%, 20 \%, 40 \%, 20 \%$, and $10 \%$, respectively (Figure 1). A change from one category to another is considered a significant change in the child's school achievement (e.g. high level of performance to satisfactory level of performance). This endpoint will be analyzed using an ordinal regression model (details are given in Additional file 1) $[53,54]$. The primary endpoint of $\mathrm{VO}_{2 \max }$ in the intervention and control groups is determined by comparing the scores at diagnosis to the scores at one year after the cessation of treatment. This is done using an independent samples t-test. Additional analyses using the four study time points (diagnosis, 3 months after diagnosis, 6 months after diagnosis, and one year after cessation of treatment) will also be performed. Means and 95\% confidence limits for the intervention and control groups will be computed at each time point using linear mixed models and the trajectories will be 


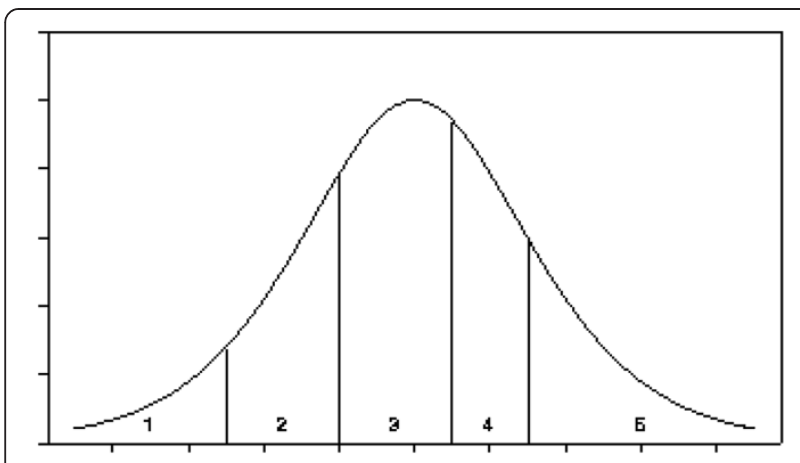

Figure 1 The structure of the ordinal regression model.

analyzed. A similar method will be used for the secondary endpoints but using only three time points for the questionnaire data. Linear mixed models will be used for each subscale of the PedsQL Core, the SDQ, the RCADS, Resilience, and the LDQ, and for the Andersen test, the timed Up-and-Go test, and the hand strength measurement. For the count data (the sit-to-stand test and the flamingo balance test), Poisson regression models with random effects will be used. Because the study is not randomized all comparisons of the two groups will be controlled for diagnosis. If any other variables are found to be unevenly distributed across the two groups additional analyses controlling for the effects of these variables will also be considered.

\section{Statistical considerations II and sample size calculations}

This study has two primary endpoints therefore the type I error is set at 0.025 in the sample size calculations. For the primary endpoint in the educational intervention the ordinal regression model with a power of 0.90 outlined in Additional file 1 will to be able to detect a shift in the ordinal rating of approximately $1 / 2$ a point, yielding a marginal frequency distribution for the five categories of $6 \%, 13 \%, 32 \%, 25 \%, 24 \%$. Details are provided in Additional file 1.

The primary endpoint of the physical and social activity study is based on the power calculation derived from a pilot study [25] that found a baseline $\mathrm{VO}_{2 \max }$ of $24.3 \mathrm{ml} / \mathrm{min} / \mathrm{kg}$ (SD 5.9) among children with acute lymphoblastic leukemia. If 120 children with cancer are included in both the intervention and control group, if the standard deviation of the change scores is 5.3 in both groups, and we use a significance level of 0.025 , we will have a power of 0.90 to detect a $10 \%$ increase in the intervention group one year after cessation of treatment.

\section{Ethical approval}

Information on the subjects is protected according to the Processing of Personal Data and Health Act. The Danish Data Protection Agency (file. 2007-58-0015/ nr.30-0734) and the Regional Ethics Committee for the Capital Region (file. H 3-2012-105) approved the project, and the project complies with the Helsinki II declaration. In addition, the study is registered at Clinical Trials.gov (file. NCT01772849 and NCT01772862). Following oral and written information, parental, guardian and child's (children above 15 year) written informed consent was obtained.

\section{Discussion}

RESPECT is based on experience from an unpublished feasibility period, a theoretical framework, and earlier intervention studies of children with cancer, but reliable evidence on rehabilitation in children with cancer is lacking. RESPECT is the first nationwide study with sufficient power to reliably test the impact of a combined multimodal intervention program during treatment. The strength of this study is the combination of educational, psychosocial, and physical components, and the study has a more interactionistic and comprehensive perspective on rehabilitation than most of the previous studies.

Although a randomized trial would be optimal from a scientific point of view, it is unrealistic because the psychological, social, and ethical aspects mean only some of the children at the same unit will have ambassadors.

Another methodological consideration is the intervention starting at diagnosis. Starting an intervention study during the course of very toxic and intensive treatment causes logistical challenges related to the disease and the side effects of the treatment. At the time of diagnosis, families are very vulnerable and stressed, and there is a potential risk of declining participation. However, the feasibility period suggested a participation rate above $90 \%$. Furthermore, the focus on rehabilitation may ameliorate the psychosocial burden on the child. The inclusion of two ambassadors from the child's school class is unique in childhood cancer rehabilitation, but also carries potential ethical concerns related to the emotional stress and school performance of the ambassadors. The feasibility period indicated that we will be able to allocate two suitable ambassadors to more than $95 \%$ of patients, and that they will be able to cope with the challenges linked to their participation. To supervise this group we have an ambassador counsel chaired by a senior child psychologist, who is not involved in the daily operation of the project. Furthermore, an ambassador manual describes the selection procedures, safety measures, and intervention possibilities, to optimize identification and psychosocial monitoring. Before being accepted as an ambassador, and after every visit to the hospital, the ambassadors are screened and have clear follow-up plans arranged. Any adverse events and complications are continuously monitored and recorded. 
Although physical exercise may cause sore muscles and stress injuries, there are no reports on such injuries in the existing literature on physical training of children with cancer. Another consideration is the level of intensity to which we exercise and test the children with cancer, from diagnosis and during treatment. Studies among children with heart diseases show that exercise training from low to high intensity is feasible and tolerable for seriously ill children [55] and it may have a positive effect on side effects such as fatigue [56]. While the primary endpoint of the physical intervention is not well documented for this study group, it is the gold standard in healthy children and a common outcome measurement in older cancer patients. The physical activity is adjusted to the treatment intensity and duration as well as the general condition of the child with cancer. During the physical intervention we monitor and assess adverse events.

We believe that RESPECT will contribute vital knowledge to the treatment and rehabilitation of children with cancer as well as other children hospitalized with long-term illness.

\section{Conclusion}

This nationwide intervention study will have the power to reliably test the impact of a comprehensive combined educational, physical, and social intervention program on the recovery and rehabilitation of childhood and adolescent patients with cancer. It is the first study to examine the effect of an early rehabilitation program including the involvement of healthy classmates as ambassadors to help reproduce a normal everyday life for children undergoing cancer treatment in hospital.

\section{Additional file}

Additional file 1: The ordinal regression model used for analysis of the educational primary endpoint $[53,54]$.

\section{Competing interests}

The authors declare that they have no competing interests.

\begin{abstract}
Authors' contributions
The author LA is scientific director of the CIRE rehabilitation program. KS is scientific director, and HBL is the main project coordinator of the RESPECT project. $T$ is responsible for the physical activity intervention together with LBO, LFT and BS. ASH is responsible for the educational intervention program together with VS and NH. SAM is responsible for the ambassador safety. $\mathrm{HH}$ and PSW are responsible for the recruitment and inclusion of participants in the West Danish control group. KVA, CH, CJ and MM are all members if the program development and scientific board of the RESPECT project. KBC are responsible for the statistical analysis. All authors read and approved the final manuscript.
\end{abstract}

\section{Acknowledgements}

The study is supported by grants from The Danish Cancer Society and The Novo Nordisk Foundation, and the Danish Children's Cancer Foundation.
We would like to acknowledge the contribution of MD Steen Rosthøj, Pediatric Department, Aarhus University Hospital and Aalborg Hospital, Denmark, who recruited patients in northern Denmark.

\section{Author details}

'Department of Pediatrics and Adolescent Medicine, Copenhagen University Hospital Rigshospitalet, Blegdamsvej 9, 2100 Copenhagen, Denmark. ${ }^{2}$ Faculty of Health Sciences, The University of Copenhagen, Blegdamsvej 2b, 2100 Copenhagen, Denmark. ${ }^{3}$ Department of Public Health and Faculty of Health Sciences, The University Hospitals Centre for Nursing and Care Research (UCSF), Copenhagen University Hospital Rigshospitalet, Ryesgade 27, 2200 Copenhagen, Denmark. ${ }^{4}$ Institute for Sport Sciences and Clinical Biomechanics, The University of Southern Denmark, Campusvej 55, 5230 Odense M, Denmark. ${ }^{5}$ Department of Biostatistics, The University of Copenhagen, Øster Farimagsgade 5, P.O. Box 2099, 1014 Copenhagen, Denmark. ${ }^{6}$ Department of Pediatrics, Aarhus University Hospital, Skejby, Brendstrupgårdsvej 100, 8200 Aarhus N, Denmark. ${ }^{7}$ Øster Farimagsgade Skole, Øster Farimagsgade 41, 2100 Copenhagen $\varnothing$, Denmark. ${ }^{8}$ Department of Oncology, Copenhagen University Hospital Rigshospitalet, Blegdamsvej 9, 2100 Copenhagen $\varnothing$, Denmark. ${ }^{9}$ Head Survivorship Department, The Danish Cancer Society Research Center, Strandboulevarden 49, 2100 Copenhagen, Denmark. ${ }^{10}$ Department of Education, Aarhus University, Campus Emdrup, Tuborgvej 164, 2400 Copenhagen, Denmark. ${ }^{11}$ Department of Occupational Therapy and Physiotherapy, Copenhagen University Hospital Rigshospitalet, Blegdamsvej 9, 2100 Copenhagen, Denmark. ${ }^{12}$ Department of Nutrition, Exercise and Sports, The University of Copenhagen, Nørre Allé 51-55, 2200 Copenhagen N, Denmark. ${ }^{13}$ Department of Pediatric Hematology and Oncology, The H. C. Andersen Children's Hospital, Odense University Hospital, Sdr. Boulevard 29, 5000 Odense C, Denmark.

Received: 14 July 2013 Accepted: 24 October 2013

Published: 14 November 2013

\section{References}

1. Brown PD, Olsen JH, Hertz H, Carstensen B, Bautz A: Survival after childhood cancer in Denmark 1943-1987: a population-based study. Ugeskr Laeger 1996, 158:773-778.

2. Gatta G, Zigon G, Capocaccia R, Coebergh JW, Desandes E, Kaatsch P, Pastore G, Peris-Bonet R, Stiller CA, The EUROCARE Working Group I: Survival of European children and young adults with cancer diagnosed 1995-2002. Eur J Cancer 2009, 45:992-1005.

3. Smith MA, Seibel NL, Altekruse SF, Ries LA, Melbert DL, O'Leary M, Smith FO, Reaman GHL: Outcomes for children and adolescents with cancer: challenges for the twenty-first century. J Clin Oncol 2010, 28:2625-2634.

4. Lund LW, Schmiegelow K, Rechnitzer C, Johansen C: A systematic review of studies on psychosocial late effects of childhood cancer: structures of society and methodological pitfalls may challenge the conclusions. Pediatr Blood Cancer 2011, 56:532-543.

5. Oeffinger KC, Mertens AC, Sklar CA, Kawashima T, Hudson MM, Meadows AT, Friedman DL, Marina N, Hobbie W, Kadan-Lottick NS, Schwartz CL, Leisenring W, Robison LL: Chronic health conditions in adult survivors of childhood cancer. N Engl J Med 2006, 355:1572-1582.

6. Vannatta K, Gerhardt CA, Wells RJ, Noll RB: Intensity of CNS treatment for pediatric cancer: prediction of social outcomes in survivors. Pediatr Blood Cancer 2007, 49:716-722.

7. Charlton A, Larcombe IJ, Meller ST, Morris Jones PH, Mott MG, Potton MW, Tranmer MD, Walker JJP: Absence from school related to cancer and other chronic conditions. Arch Dis Child 1991, 66:1217-1222.

8. Barrera M, Shaw AK, Speechley KN, Maunsell E, Pogany L: Educational and social late effects of childhood cancer and related clinical, personal, and familial characteristics. Cancer 2005, 104:1751-1760.

9. Suzuki LK, Kato PM: Psychosocial support for patients in pediatric oncology: the influences of parents, schools, peers, and technology. J Pediatr Oncol Nurs 2003, 20:159-174.

10. Adamoli L, Deasy-Spinetta P, Corbetta A, Jankovic M, Lia R, Locati A, Fraschini D, Masera G, Spinetta JJ: School functioning for the child with leukemia in continuous first remission: screening high-risk children. Pediatr Hematol Oncol 1997, 14:121-131.

11. Gregory K, Parker L, Craft AW: Returning to primary school after treatment for cancer. Pediatr Hematol Oncol 1994, 11:105-109. 
12. Gurney JG, Krull KR, Kadan-Lottick N, Nicholson HS, Nathan PC, Zebrack B, Tersak JM, Ness KN: Social outcomes in the childhood cancer survivor study cohort. J Clin Oncol 2009, 27:2390-2395.

13. Mitby PA, Robison LL, Whitton JA, Zevon MA, Gibbs IC, Tersak JM, Meadows AT, Stovall M, Zeltzer LK, Mertens AC: Utilization of special education services and educational attainment among long-term survivors of childhood cancer. Cancer 2003, 97:1115-1126.

14. Gerhardt CA, Dixon M, Miller K, Vannatta K, Valerius KS, Correll J, Noll RB: Educational and occupational outcomes among survivors of childhood cancer during the transition to emerging adulthood. J Dev Behav Pediatr 2007, 28:448-455

15. Gerhardt CA, Vannatta K, Valerius KS, Correll J, Noll RB: Social and romantic outcomes in emerging adulthood among survivors of childhood cancer. $J$ Adolescent Health 2007, 40:462.e9-462.e15.

16. Glaser AW, U CL, Abdul Rashid NF, Walker DA: School behaviour and health status after central nervous system tumours in childhood. Brit J Cancer 1997, 76:643-650.

17. Koch SV, Kejs AM, Engholm G, Johansen C, Schmiegelow K: Educational attainment among survivors of childhood cancer: a population-based cohort study in Denmark. Brit J Cancer 2004, 91:923-928.

18. Harila-Saari AH, Lähteenmäki PM, Pukkala E, Kyyrönen P, Lanning M, Sankila R: Scholastic achievements of childhood leukemia patients: a nationwide, register-based study. J Clin Oncol 2007, 25:3518-3524.

19. Lähteenmäki PM, Huostila J, Hinkka S, Salmi TT: Childhood cancer patients at school. Eur J Cancer 2002, 38:1227-1240.

20. Ott JS, Webb TE, Anderson CA, Kastelic JE, Krill CE Jr: Childhood cancer and vulnerability for significant academic underachievement. J Learn Disabil 1982, 15:363-365.

21. Katz ER, Varm JW, Rubenstein CL, Blew A, Hubert N: Teacher, parent, and child evaluative ratings of a school reintegration intervention for children with newly diagnosed cancer. Child Health Care 1992, 21:69-75.

22. Braam Kl, Van Dijk EM, Veening MA, Bierings MB, Merks JH, Grootenhuis MA, Chinapaw MJM, Sinnem G, Takken T, HuisMan J, Kaspers GJL, Broeder ED: Design of the quality of life in motion (QLIM) study: a randomized controlled trial to evaluate the effectiveness and cost-effectiveness of a combined physical exercise and psychosocial training program to improve physical fitness in children with cancer. BMC Cancer 2010, 10:624.

23. Huang TT, Ness KK: Exercise interventions in children with cancer: a review. Int J Pediatr 2011, 2011:Article ID 461512

24. Winter C, Müller C, Hoffmann C, Boos J, Rosenbaum D: Physical activity and childhood cancer. Pediatr Blood Cancer 2010, 54:501-510.

25. San Juan AF, Fleck SJ, Chamorro-Vina C, Maté-Mũnoz JL, Moral S, Perez M, et al: Effects of an intrahospital exercise program intervention for children with leukemia. Med Sci Sport Exer 2007, 39:13-21.

26. San Juan AF, Fleck SJ, Chamorro-Vina C, Maté-Mũnoz JL, Moral S, GarciaCastro J, Ramirez M, Madero L, Lucia A: Early-phase adaptations to intrahospital training in strength and functional mobility of children with leukemia. J Strength Cond Res 2007, 21:173-177.

27. Stam H, Grootenhuis MA, Last BF: The course of life of survivors of childhood cancer. Psycho-oncol 2005, 14:227-238.

28. DuHamel KN, Redd WH, Vickberg SM: Behavioral interventions in the diagnosis, treatment and rehabilitation of children with cancer. Acta Oncol 1999, 38:719-734.

29. Lancashire ER, Frobisher C, Reulen RC, Winter DL, Glaser A, Hawkins MM: Educational attainment among adult survivors of childhood cancer in Great Britain: a population-based cohort study. J Natl Cancer Inst 2010, 102:254-270.

30. Maurice-Stam H, Silberbusch LM, Last BF, Grootenhuis MA: Evaluation of a psycho-educational group intervention for children treated for cancer: a descriptive pilot study. Psycho-oncol 2009, 18:762-766

31. Mazurek Melnyk B: Intervention studies involving parents of hospitalized young children: an analysis of the past and future recommendations. $J$ Pediatr Nurs 2000, 15:4-13.

32. Varni JW, Katz ER, Colegrove $R$ Jr, Dolgin M: The impact of social skills training on the adjustment of children with newly diagnosed cancer. J Pediatr Psychol 1993, 18:751-767.

33. Goffman E: The presentation of self in everyday life. New York: Doubleday; 1959.

34. Goffman E: Stigma: notes on the management of spoiled identity. New York: Touchstone; 1963.

35. Misztal BA: Normality and trust in Goffman's theory of interaction order. Sociol Theor 2001, 19:312-324.
36. Collins R: Stratification: a micro-macro theory of inequality. Socio/ Theor 2000, 18:17-43.

37. Scheff TJ: Shame and the social bond: a sociological theory. Sociol Theor 2000, 18:84-99.

38. Simovska V: Learning by interaction: learning about health through participation and action-the health promoting schools perspective. Copenhagen: Danmarks Lærerhøjskole; 2005.

39. Godfrey S, Davies CTM, Wozniak E, Barnes CA: Cardio-respiratory response to exercise in normal children. Clin Sci 1971, 40:419-431.

40. Varni JW, Burwinkle TM, Katz ER, Meeske K, Dickinson P: The PedsQL ${ }^{\mathrm{TM}}$ in pediatric cancer: reliability and validity of the pediatric quality of life inventory ${ }^{\mathrm{TM}}$ generic core scales, multidimensional fatigue scale, and cancer module. Cancer 2002, 94:2090-2106.

41. Goodman R: The strengths and difficulties questionnaire: a research note. J Child Psychol Psyc 1997, 38:581-586.

42. Chorpita BF, Yim L, Moffitt C, Umemoto LA, Francis SE: Assessment of symptoms of DSM-IV anxiety and depression in children: a revised child anxiety and depression scale. Behav Res Ther 2000, 38:835-855.

43. Friborg $\mathrm{O}$, Hjemdal $\mathrm{O}$, Rosenvinge $\mathrm{JH}$, Martinussen $\mathrm{M}$ : A new rating scale for adult resilience: what are the central protective resources behind healthy adjustment? Int J Meth Psych Res 2003, 12:65-76.

44. Asher SR, Wheeler VA: Children's loneliness: a comparison of rejected and neglected peer status. J Consult Clin Psych 1985, 53:500-505.

45. Horowitz M, Wilner N, Alvarez W: Impact of event scale: a measure of subjective stress. Psychosom Med 1979, 41:209-218.

46. Bulowski W, Hoza B: Popularity and friendship: issues, measurement, and outcome. In Peer relationships in child development. Edited by Berndt TJ, Ladd GW. New York: Wiley; 1989:15-45.

47. Andersen LB, Andersen TE, Andersen E, Anderssen SA: An intermittent running test to estimate maximal oxygen uptake: the Andersen test. J Sport Med Phys Fit 2008, 48:434-437.

48. Shephard RJ, Allen C, Benade AJ, Davies CT, Di Prampero PE, Hedman R, Merriman JE, Myhre K, Simmons R: The maximum oxygen intake: an international reference standard of cardiorespiratory fitness. $B$ World Health Organ 1968, 38:757-764.

49. Podsiadlo D, Richardson S: The timed "Up \& Go": a test of basic functional mobility for frail elderly persons. J Am Geriatr Soc 1991, 39:142-148.

50. Bohannon RW: Sit-to-stand test for measuring performance of lower extremity muscles. Percept Motor Skills 1995, 80:163-166.

51. Abizanda P, Navarro JL, Garcia-Tomás MI, López-Jiménez E, Martínez-Sánchez E, Paterna G: Validity and usefulness of hand-held dynamometry for measuring muscle strength in community-dwelling older persons. Arch Gerontol Geriat 2012, 54:21-27.

52. Deforche B, Lefevre J, De Bourdeauhuij I, Hills AP, Duquet W, Bouckaert J: Physical fitness and physical activity in obese and nonobese Flemish youth. Obesity 2003, 11:434-441.

53. McCullagh P: Regression models for ordinal data. J Roy Stat Soc B 1980 42:109-142

54. Muthén B: A general structural equation model with dichotomous, ordered categorical, and continous latent variable indicators. Psychometrika 1984, 49:115-132.

55. Rognmo Ø, Hetland E, Helgerud J, Hoff J, Slørdahl SA: High intensity aerobic interval exercise is superior to moderate intensity exercise for increasing aerobic capacity in patients with coronary artery disease. Eur J Cardiov Prev R 2004, 11:216-222.

56. Galvão DA, Newton RU: Review of exercise intervention studies in cancer patients. J Clin Oncol 2005, 23:899-909.

doi:10.1186/1471-2407-13-544

Cite this article as: Thorsteinsson et al.: Study protocol: rehabilitation including social and physical activity and education in children and teenagers with cancer (RESPECT). BMC Cancer 2013 13:544. 